\title{
Hemophagocytic lymphohistiocytosis in a patient with glioblastoma: a case report
}

\author{
Vaibhav Kumar ${ }^{1}$, Patrick J Eulitt ${ }^{1}$, Ana Bermudez ${ }^{2}$ \& Simon Khagi*,1 \\ ${ }^{1}$ Division of Hematology \& Oncology, University of North Carolina at Chapel Hill, Chapel Hill, NC 27599, USA \\ ${ }^{2}$ Division of Internal Medicine, University of North Carolina at Chapel Hill, Chapel Hill, NC 27599, USA \\ *Author for correspondence: skhagi@med.unc.edu
}

\section{Practice points}

- Hemophagocytic lymphohistiocytosis (HLH) among adults with solid organ malignancies is usually secondary to a precipitating infective etiology such as Epstein-Barr viremia.

- Patients are often critically ill at the time of presentation and a high index of suspicion is needed for making the diagnosis.

- Readers should be aware of the HLH-2004 diagnostic criteria, which is a combination of clinical, biochemical and pathologic variables that are required to make the diagnosis.

- The absence of hemophagocytosis does not exclude the diagnosis of HLH.

- Rituximab, a monoclonal antibody against CD20, has been shown to be effective in managing Epstein-Barr viremia associated HLH.

- Secondary HLH has a high mortality rate and prompt initiation of therapies and multidisciplinary discussion with oncologists, hematologists and infectious disease specialists is highly encouraged.

Adult onset hemophagocytic lymphohistiocytosis (HLH) is a rare condition, usually secondary to either a precipitating infective or hematologic malignancy. We present a case of Epstein-Barr virus associated HLH in a 55-year-old female receiving treatment for a glioblastoma (GBM). It is possible that HLH is under recognized, as patients with GBM often have features of a nonspecific systemic inflammatory response syndrome, multiorgan failure and cognitive decline. A high index of suspicion and increased awareness can help improve timeliness of diagnosis. Therapeutically, Epstein-Barr virus associated HLH in patients with solid organ malignancy poses significant challenges. An individualized, multidisciplinary approach is essential when managing adult-onset $\mathrm{HLH}$ and providers will need to be mindful of the high mortality rate despite treatment.

First draft submitted: 3 July 2019; Accepted for publication: 9 October 2019; Published online:

28 November 2019

Keywords: Epstein-Barr virus $\bullet$ glioblastoma $\bullet$ hemophagocytic lymphohistiocytosis $\bullet$ hyperferritinemia $\bullet$ pancytopenia $\bullet$ splenomegaly $\bullet$ temozolomide

Hemophagocytic lymphohistiocytosis (HLH) is a rare disorder with a high mortality rate, the pathophysiologic hallmark being an overactive and ineffective immune system [1]. HLH was initially described, and is best recognized, in the pediatric population; however, there is increasing recognition of this disorder among adults [2]. HLH is categorized as either primary (familial) or, more commonly in adults, secondary to an underlying infective, autoimmune, metabolic or malignant process [3]. In the secondary setting, prompt recognition is essential as the 1 -month mortality is estimated at $20 \%$ [4] and the median overall survival among patients with an underlying malignancy is 1.4 months [5].

In a systematic review of secondary causes of 2197 patients with HLH, an underlying hematologic malignancy was found in $45 \%(\mathrm{n}=981)$ with only $1.5 \%(\mathrm{n}=32)$ having an underlying solid organ malignancy [2]. We describe a case of HLH in a patient undergoing active treatment for glioblastoma (GBM) who had evidence of increasing frequency of opportunistic infections in the setting of persistent treatment-related lymphopenia. To the best of our knowledge, this is the first case report that describes the diagnosis of HLH in a patient with GBM and its subsequent management, with the only other case being noted in a single institution case series of HLH with 


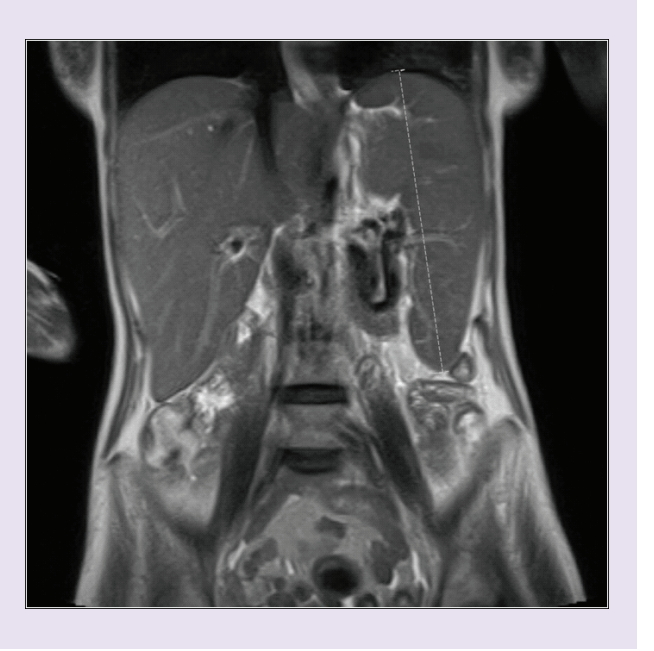

Figure 1. Magnetic resonance image of abdomen without contrast coronal cross-section with spleen measuring $19 \mathrm{~cm}$.

no further clinical details available [6]. Increasing awareness of the diagnostic criterion can improve identification, reporting and ultimately allow improved individualized decision making at a patient level.

\section{Case}

Our patient is an otherwise healthy 55-year-old female who initially presented with right-sided weakness and expressive aphasia to an outside hospital. Imaging findings revealed a left parietal-thalamic lesion, suspicious for a high-grade glioma. She underwent a subtotal resection and pathology revealed a GBM. Her molecular markers suggested an aggressive phenotype (i.e., an unmethylated $M G M T$ promotor and without an isocitrate dehydrogenase mutation). At the time of diagnosis, she had a Karnofsky performance status of 60, entirely attributed to her malignancy, and was slightly underweight with a BMI of $17 \mathrm{~kg} / \mathrm{m}^{2}$. She subsequently started chemoradiation, but her course was complicated by obstructive hydrocephalus requiring implantation of an intraventricular peritoneal shunt. Despite standard therapy, her GBM continued to progress. She was started on bevacizumab and metronomic (daily low dose) temozolomide as second-line treatment.

Approximately 6 weeks prior to her most recent hospitalization, she was evaluated for hypoxemic respiratory failure. Her initial infectious workup included blood and respiratory cultures as well as a respiratory viral panel, all of which were negative. She did not improve on empiric antibiotics and was thought to have Pneumocystis jiroveci pneumonia (PJP) given her improvement after initiation of steroids and trimethoprim with sulfamethoxazole (TMP-SMX). After starting TMP-SMX, her absolute neutrophil count began to drop. She was subsequently readmitted with malaise, cough and was found to have an absolute neutrophil count of $800 \times 10^{6} / 1$. Notably, her absolute lymphocyte count remained persistently low since the initiation of chemoradiation. She was readmitted 3 weeks prior to her most recent hospitalization for increased shortness of breath and during admission tested positive for coronavirus. Her TMP-SMX was changed to dapsone in order to continue prophylactic PJP coverage. She was seen for an outpatient oncology office visit 1 week prior to admission, at which time her respiratory status had returned to baseline with a Karnofsky performance status of 70 . Her absolute neutrophil count began to recover; however, her absolute lymphocyte count remained below $200 \times 10^{6} / 1$.

She was readmitted with worsening shortness of breath and confusion and was found to be hypotensive, tachycardic and hypoxemic. Laboratory studies revealed worsening pancytopenia and elevation in liver enzymes. Abdominal ultrasound and MRI demonstrated a heterogeneous liver with coarse echotexture and splenomegaly (Figure 1) with no significant antecedent alcohol use or liver disease. MRI of the brain revealed a stable appearing GBM. She was started on fluids and antibiotics. Liver injury was initially thought to be drug induced in the setting of bevacizumab, TMP-SMX, temozolomide and dapsone. On routine type and screen, she was noted to have new warm autoantibodies and a positive direct-antiglobulin test, concerning for hemolytic anemia, although her total indirect and direct bilirubin were not markedly elevated and could be alternatively explained by her liver injury ( 0.9 and $1.4 \mathrm{mg} / \mathrm{dl}$, respectively). Fibrinogen levels were also noted to be low, with elevated d-dimer $(820 \mathrm{ng} / \mathrm{ml})$ and mild coagulopathy (PT 16.8 s, aPTT 48.2 s), all consistent with liver injury. Viral studies revealed an Epstein-Barr virus (EBV) quantitative load of 175,266 international units per $\mathrm{ml}(\mathrm{IU} / \mathrm{ml})$. She had no known history of EBV 
Table 1. Patient requires greater than or equal to 5 of 8 diagnostic criteria.

\begin{tabular}{|c|c|}
\hline \multicolumn{2}{|l|}{ Diagnostic criteria } \\
\hline 1. Fever ${ }^{\dagger}$ & Temperature $>38.5^{\circ} \mathrm{C}$ for $>7$ days \\
\hline 2. Splenomegaly ${ }^{\dagger}$ & Spleen tip palpated $>3 \mathrm{~cm}$ below left costal margin \\
\hline 3. Cytopenia: & $\geq 2$ lineages \\
\hline Hemoglobin $^{\dagger}$ & $<90 \mathrm{~g} / \mathrm{l}$ (neonates $<100 \mathrm{~g} / \mathrm{l}$ ) \\
\hline Platelets ${ }^{\dagger}$ & $<100 \times 10^{9} / 1$ \\
\hline Neutrophil ${ }^{\dagger}$ & $<1 \times 10^{9} / 1$ \\
\hline 4. Hyperferritinemia ${ }^{\dagger}$ & $>500 \mu \mathrm{g} / \mathrm{l}$ \\
\hline 5. Hypofibrinogenemia ${ }^{\dagger}$ or hypertriglyceridemia ${ }^{\dagger}$ & $<1.5 \mathrm{~g} / \mathrm{l}$, or $>3 \mathrm{mmol} / \mathrm{l}$ \\
\hline 6. Elevated soluble $\mathrm{CD} 25^{\dagger}$ & $>2400 \mathrm{U} / \mathrm{ml}$ \\
\hline 7. Hemophagocytosis & Bone marrow, spleen, liver, lymph node or other tissues \\
\hline \multicolumn{2}{|l|}{ 8. Reduced or absent NK cytotoxicity } \\
\hline \multicolumn{2}{|l|}{ Supportive evidence } \\
\hline \multicolumn{2}{|l|}{ Elevated transaminases and bilirubin ${ }^{\dagger}$} \\
\hline \multicolumn{2}{|l|}{ Elevated lactate dehydrogenase $^{\dagger}$} \\
\hline \multicolumn{2}{|l|}{ Elevated d-dimers ${ }^{\dagger}$} \\
\hline \multicolumn{2}{|l|}{ Elevated cerebrospinal fluid cells and/or protein } \\
\hline
\end{tabular}

infection. Given her precipitous clinical decline, liver dysfunction, pancytopenia and hypofibrinogenemia, workup for HLH was initiated. She was found to have a ferritin level of $27,100 \mathrm{ng} / \mathrm{ml}$, a fasting hypertriglyceridemia of $940 \mathrm{mg} / \mathrm{dl}$ and an elevated soluble IL-2 receptor at 14,070 units/ml. This made HLH a likely diagnosis (Table 1). A bone marrow biopsy was not attempted given her clinical condition.

\section{Treatment}

She was stared on methylprednisolone and intravenous immunoglobulins with little effect. She required multiple transfusions of blood, platelets and cryoprecipitate for disseminated intravascular coagulation. Rituximab was initiated, followed by daily anakinra. There was some notable response in hematologic parameters, and she required less transfusions (Figure 2). Unfortunately, the patient's condition continued to deteriorate. She became oliguric and an echocardiogram revealed a Takotsubo-pattern cardiomyopathy. She went on to develop pancreatitis due to hypertriglyceridemia. Given the extensive multiorgan failure and her family's wishes not to initiate ventilator support or dialysis, the patient passed away shortly after de-escalating her care.

\section{Discussion}

Patients with secondary HLH have a diverse presentation, making the diagnosis difficult to obtain. The most frequently used diagnostic criterion (HLH-2004) was developed in the pediatric population to aid enrollment into clinical trials [7]. The HLH-2004 is now commonly used in the adult population and usually greater than or equal to five of the eight diagnostic criteria is supportive of the diagnosis in adults (Table 1). In a retrospective evaluation of the diagnostic performance of the HLH-2004 criteria across pediatric and adult populations, Debaugnies et al. demonstrated a sensitivity of $55 \%$ and specificity of $100 \%$ in adults, who had five or more of the diagnostic criteria at initial presentation [8]. Notably, the authors excluded the criteria of reduced NK cytotoxicity and elevated soluble IL-2 (sCD25) from their analysis. The role of evaluating NK-cell activity is best described in the pediatric population and is not usually done in adults [1]. Our patient had an sCD25 level of $>10,000 \mathrm{u} / \mathrm{ml}$, which in a cohort of patients with prevalence of $\mathrm{HLH}$ of $49 \%$ yields a positive predictive value of $85 \%$, a sensitivity of $45 \%$ and specificity of $93 \%$ [9]. Although hemophagocytosis is a hallmark pathological feature of HLH, our patient did not undergo a bone marrow biopsy due to their critical illness. Hemophagocytosis is neither sensitive or specific [1]; Gupta et al. have previously demonstrated in pediatric patients that the median time to diagnosis of HLH from the initial bone marrow aspirate is 0 day [10], suggesting that diagnosis is often made prior to the outcome of the bone marrow biopsy. Given the high index of suspicion for HLH in our patient, a bone marrow aspirate with no evidence of hemophagocytosis would not have altered our initial treatment approach. 


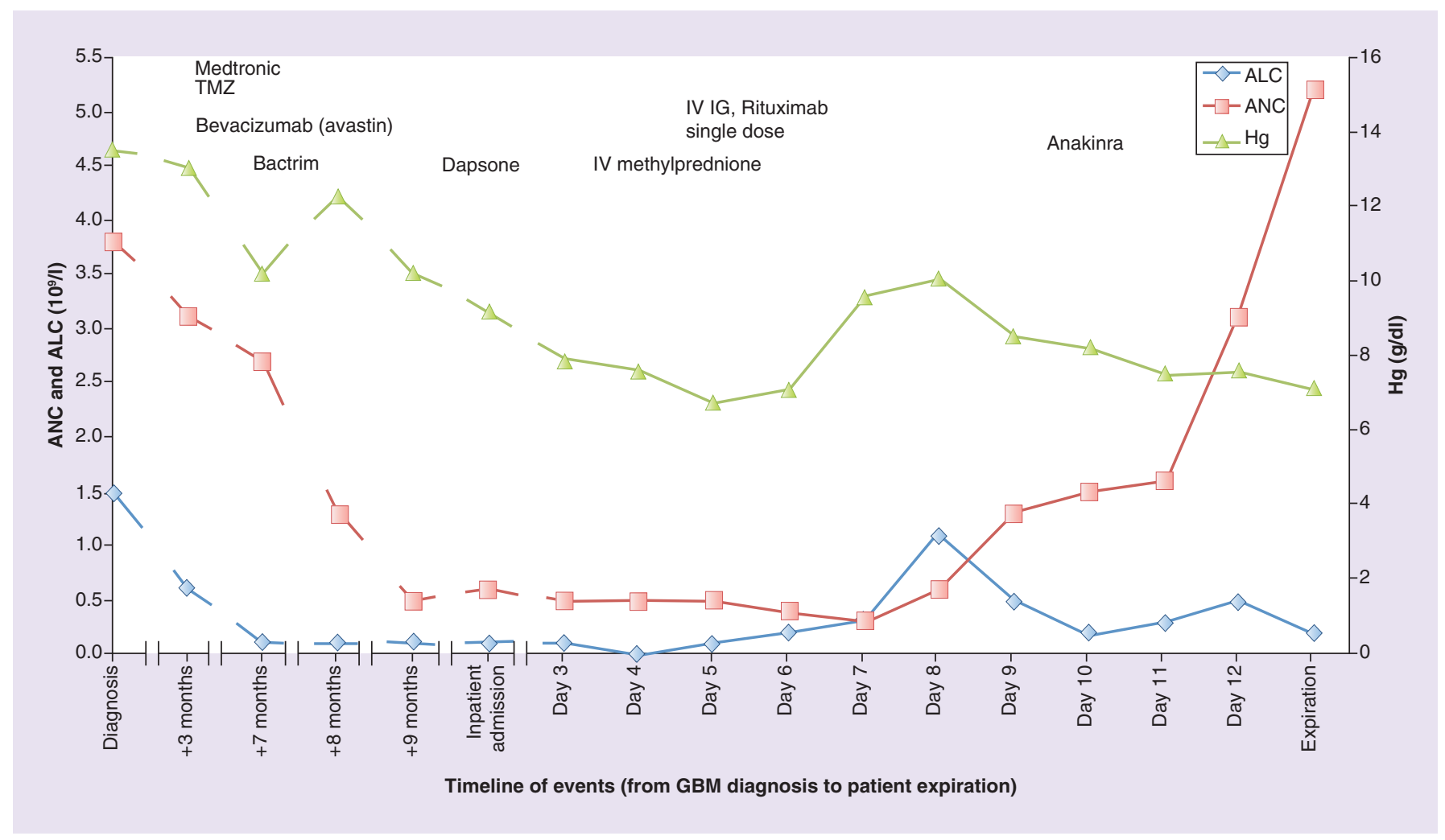

Figure 2. Treatment timeline and response to hematologic parameters. The patient had persistent and severe lymphocytopenia following a diagnosis of glioblastoma.

ALC: Absolute lymphocyte count; ANC: Absolute neutrophil count; GBM: Glioblastoma; IVIg: Intravenous immunoglobulin; Hg: Hemoglobin; TMZ: Temozolomide.

Notably, our patient was receiving standard therapy for her GBM with temozolomide, an oral alkylating agent, which is commonly associated with lymphopenias and an increased risk of opportunistic infections [11]. Due to the concerns for disease progression, the patient had also received significant doses of corticosteroids and bevacizumab, a monoclonal antibody which reduced angiogenesis through inhibition of VEGF, both commonly used in the management of GBM, but also associated with immunosuppression and infective complications [12]. Her HIV testing was negative, however, she had severe lymphopenia with a CD4 count of $24 \times 10^{6}$ and had microbiologic evidence of EBV infection in addition to recently being treated for PJP. We suspect that the likely trigger for HLH in our patient was reactivation of EBV in the setting of immunosuppression in conjunction with temozolomide therapy in the setting of her GBM.

EBV is most commonly associated infective trigger that can result in the diagnosis of $\mathrm{HLH}$ [2], and is associated with poor outcomes [13]. There is great geographic variability in the diagnosis of EBV-associated HLH, with the greatest experience being driven from Asia. Etoposide and dexamethasone form the mainstay of the initial treatment, including patients with EBV-associated HLH [14], and this has been confirmed in the long-term analysis of the HLH-2004 study [15]. The administration of etoposide in our patient was difficult due to the severe hepatic dysfunction, thus we chose our initial therapy with corticosteroids and intravenous immunoglobulins [16], with an intent to start etoposide within a 4-week window [17]. Rituximab, a monoclonal antibody against CD20, has been shown to be effective in reducing symptoms, inflammation and EBV viral load in patients with EBV-associated HLH [18]. Our patient continued to deteriorate, and the therapeutic decision was to start anti-IL-1 therapy, anakinra versus the anti-CD52 targeting monoclonal antibody, alemtuzumab. Both agents have been used in the refractory setting $[19,20]$. The management of HLH in her case posed several diagnostic and therapeutic challenges. Despite making a diagnosis, the toxicity profile of the proposed interventions was considerable. Additionally, the patient has the competing risks of mortality from her underlying malignancy, severe infective complications and multiorgan failure. With increasing use of immunosuppressive and cytotoxic regimens for the management of 
advanced malignancies, it is likely that there will be increasing recognition of HLH in patients with solid organ malignancy and increased awareness and further research on best therapeutic approach to reduce the high mortality rate. Additionally, patients with high-grade gliomas treated with temozolomide should be monitored for signs of HLH.

\section{Conclusion}

$\mathrm{HLH}$ is a rare and highly morbid condition in adults. We present the first case of a patient who developed EBV-associated HLH following therapy for underlying GBM. In addition to a high index of suspicion for the diagnosis, especially in patients with solid organ malignancy, a carefully considered approach will be needed when extrapolating the therapeutic approaches, the evidence base is largely derived from the pediatric population.

\section{Financial \& competing interests disclosure}

The authors have no relevant affiliations or financial involvement with any organization or entity with a financial interest in or financial conflict with the subject matter or materials discussed in the manuscript. This includes employment, consultancies, honoraria, stock ownership or options, expert testimony, grants or patents received or pending, or royalties.

VK received support from the NIH T32 training grant (5T32HL007149-43). The content is solely the responsibility of the authors and does not necessarily represent the official views of the $\mathrm{NIH}$.

No writing assistance was utilized in the production of this manuscript.

\section{Ethical conduct of research}

The authors state that they have followed the principles outlined in the Declaration of Helsinki for all human or animal experimental investigations. The authors state that they have obtained verbal and written informed consent from the patient's next of kin for the inclusion of their medical and treatment history within this case report.

\section{Open access}

This work is licensed under the Attribution-NonCommercial-NoDerivatives 4.0 Unported License. To view a copy of this license, visit http://creativecommons.org/licenses/by-nc-nd/4.0/

\section{References}

Papers of special note have been highlighted as: $\bullet$ of interest; $\bullet \bullet$ of considerable interest

1. Schram AM, Berliner N. How I treat hemophagocytic lymphohistiocytosis in the adult patient. Blood 125(19), 2908-2914 (2015).

- This is a very well written review by an international expert that discusses the diagnostic and therapeutic dilemmas in hemophagocytic lymphohistiocytosis (HLH).

2. Ramos-Casals M, Brito-Zerón P, López-Guillermo A, Khamashta MA, Bosch X. Adult haemophagocytic syndrome. Lancet 383(9927), 1503-1516 (2014).

3. Emilie JF, Abla O, Fraitag S et al. Revised classification of histiocytoses and neoplasms of the macrophage-dendritic cell lineages. Blood 127(22), 2672-2682 (2016).

4. Rivière S, Galicier L, Coppo P et al. Reactive hemophagocytic syndrome in adults: a retrospective analysis of 162 patients. Am. J. Med. 127(11), 1118-1125 (2014).

5. Parikh SA, Kapoor P, Letendre L, Kumar S, Wolanskyj AP. Prognostic factors and outcomes of adults with hemophagocytic lymphohistiocytosis. Mayo Clin. Proc. 89(4), 484-492 (2014).

6. Egues Dubuc CA, Aldasoro Cáceres V, Meneses Villalba C, Ecenarro Uriarte M, Hernando Rubio I, Belzunegui Otano J. THU0452 secondary hemophagocytic lymphohistiocytosis: a series of 11 patients. Literature Review. Ann. Rheum. Dis. 72(Suppl. 3), A317.2-A317 (2013).

7. Henter J-I, Horne A, Aricó M et al. HLH-2004: diagnostic and therapeutic guidelines for hemophagocytic lymphohistiocytosis. Pediatr. Blood Cancer 48(2), 124-131 (2007).

-. This is a highly cited international consensus for the diagnosis and approach to management of patients with HLH.

8. Debaugnies F, Mahadeb B, Demulder A et al. Performances of the $\mathrm{H}$-score for diagnosis of hemophagocytic lymphohistiocytosis in adult and pediatric patients. Am. J. Clin. Pathol. 145(6), 862-870 (2016).

- This original article compares a newly developed H-score to HLH-2004 criteria when approaching the diagnosis of HLH.

9. Hayden A, Lin M, Park S et al. Soluble interleukin-2 receptor is a sensitive diagnostic test in adult HLH. Blood Adv. 1(26), 2529-2534 (2017).

10. Gupta A, Tyrrell P, Valani R, Benseler S, Weitzman S, Abdelhaleem M. The role of the initial bone marrow aspirate in the diagnosis of hemophagocytic lymphohistiocytosis. Pediatr. Blood Cancer 51(3), 402-404 (2008). 
11. Stupp R, Mason WP, van den Bent MJ et al. Radiotherapy plus concomitant and adjuvant temozolomide for glioblastoma. N. Engl. J. Med. 352(10), 987-996 (2005).

12. Vredenburgh JJ, Cloughesy T, Samant M et al. Corticosteroid use in patients with glioblastoma at first or second relapse treated with bevacizumab in the BRAIN study. Oncologist 15(12), 1329-1334 (2010).

13. Rouphael NG, Gould C. Infections associated with haemophagocytic syndrome. Lancet Infect. Dis. 7(12), 814-822 (2007).

- This is a good review on the common infectious diseases associated with a diagnosis of HLH.

14. Imashuku S, Kuriyama K, Teramura $\mathrm{T}$ et al. Requirement for etoposide in the treatment of Epstein-Barr virus-associated hemophagocytic lymphohistiocytosis. J. Clin. Oncol. 19(10), 2665-2673 (2001).

15. Bergsten E, Horne A, Aricó M et al. Confirmed efficacy of etoposide and dexamethasone in HLH treatment: long-term results of the cooperative HLH-2004 study. Blood 130(25), 2728-2738 (2017).

16. Ishii E, Ohga S, Imashuku S et al. Nationwide survey of hemophagocytic lymphohistiocytosis in Japan. Int. J. Hematol. 86(1), 58-65 (2007).

17. Imashuku S. Treatment of Epstein-Barr virus-related hemophagocytic lymphohistiocytosis (EBV-HLH); Update 2010. J. Pediatr. Hematol. Oncol. 33(1), 35-39 (2011).

18. Chellapandian D, Das R, Zelley K et al. Treatment of Epstein Barr virus-induced haemophagocytic lymphohistiocytosis with rituximab-containing chemo-immunotherapeutic regimens. Br. J. Haematol. 162(3), 376-382 (2013).

19. Marsh RA. Epstein-Barr Virus and hemophagocytic lymphohistiocytosis. Front. Immunol. 8, 1902 (2017).

20. Marsh RA, Allen CE, McClain KL et al. Salvage therapy of refractory hemophagocytic lymphohistiocytosis with alemtuzumab. Pediatr. Blood Cancer 60(1), 101-109 (2013). 